\title{
Wasserstoff $\left(\mathrm{H}_{2}\right)$-Exhalation nach Verabreichung von Laktose, Lactulose und Xylose beim Fohlen
}

\author{
Verena Bracher, Jennifer C. Ousey, Emily Beatty, P. D. Rossdale und W.R. Allen \\ Klinik für Wiederkäuer und Pferdemedizin, Veterinärmedizinische Fakultät der Universität Zürich
}

\begin{abstract}
Zusammenfassung
Bei 10 Saugfohlen und 3 mutterlosen Fohlen im Alter zwischen 23 und 103 Tagen wurde die Wasserstoff-Exhalation nach Verabreichung von $1.0 \mathrm{~g} / \mathrm{kg} \mathrm{KM}$ Laktose, $0.5-1.0 \mathrm{~g} / \mathrm{kg} \mathrm{KM}$ Lactulose und $0.5 \mathrm{~g} / \mathrm{kg} \mathrm{KM}$ Xylose in halbstündlichen Intervallen über einen Zeitraum von 8 Stunden bestimmt. Zusätzlich wurde nach Xylose-Verabreichung die Plasma-Xylose-Konzentrationen bestimmt. Bei den Saugfohlen erfolgte die Verabreichung nach 3stündigem Fasten mit einer Nasenschlundsonde. Bei den mutterlosen Fohlen wurde die Testsubstanz morgens mit der ersten Milchersatzmahlzeit zusammen verabreicht. Ein Anstieg der $\mathrm{H}_{2}$-Exhalation nach Verabreichung der Zucker wurde bei den meisten Fohlen beobachtet, allerdings mit großen Schwankungen zwischen den einzelnen Tieren.
\end{abstract}

Schlüsselwörter: Fohlen, Wasserstoff-Exhalationstest, Xylose-Absorptionstest, Laktose, Lactulose

\begin{abstract}
Breath $\left(\mathrm{H}_{2}\right)$ excretion after administration of lactose, lactulose and xylose in foals
Ten sucking and 3 orphan foals aged 23-103 days were used to study breath hydrogen excretion after administration of $1.0 \mathrm{~g} / \mathrm{kg}$ bwt. lactose, $0.5-1.0 \mathrm{~g} / \mathrm{kg}$ bwt. lactulose and $0.5 \mathrm{~g} / \mathrm{kg}$ bwt. xylose. Breath samples were taken in $30 \mathrm{~min}$ intervals over a time period of $8 \mathrm{hrs}$ after administration. In addition, plasma xylose levels were measured in the 6 foals that received xylose, either by stomach tube (sucking foals, $n=3$ ) or added to the normal feed of millk replacer (orphan foals, $n=3$ ). Breath hydrogen excretion was observed in almost all animals after sugar administration, but high individual variations in time to the first peak and height of peak was observed.
\end{abstract}

keywords: $\quad$ foal, hydrogen breath test, xylose absorption test, lactose, lactulose

\section{Einleitung}

Durch die Bestimmung von Wasserstoff $\left(\mathrm{H}_{2}\right)$ in der Ausatmungsluft kann sowohl eine indirekte Aussage über die fermentativen Aktivitäten im Gastrointestinaltrakt von Monogastriern gemacht (Calloway et al. 1966, Levitt et al. 1968, Zentek 1992, Christl et al. 1995) als auch die orozäkale Transitzeit bestimmt werden (Abrahamsson 1988). In einer früheren Untersuchung (Bracher et al. 1995a) konnte gezeigt werden, daß Saugfohlen im Alter von 20-60 Tagen hohe $\mathrm{H}_{2}$-Konzentrationen in der Ausatmungsluft aufweisen und es wurde vermutet, daß es sich dabei um eine partielle mikrobielle Verdauung der Laktose im Dickdarmbereich handelt. Beim normalen Kleinkind wird Laktose im Dünndarm verdaut und führt deshalb nicht zu einem Anstieg der $\mathrm{H}_{2}$-Exhalation (Douwes et al. 1985). Beim frühreifen Säugling erfolgt nur eine partielle Dünndarmverdauung der Laktose und daraus resultiert eine mikrobielle Verstoffwechselung des Substrates im Zäkum und Kolon, was zu einer deutlichen $\mathrm{H}_{2}$-Exhalation führt (Kien et al. 1987). Lactulose andererseits, ein Disaccharid bestehend aus 1,4-beta-glykosierter Galaktose und Fruktose, wird nicht im Dünndarm gespalten und steht somit prinzipiell der mikrobiellen Vergärung im Zäkum und Kolon zur Verfügung. Aus diesem Grund wird
Lactulose als die klassische Testsubstanz zur Überprüfung der $\mathrm{H}_{2}$-bildenden Kapazität und zur Bestimmung der orozäkalen Transitzeit beim Menschen verwendet (Bond und Levitt 1975).

Xylose, eine Pentose, die über den Glukose-Carrier im Dünndarm resorbiert wird und als Testsubstanz für die Dünndarmfunktion des adulten Pferdes und des Fohlens verwendet wird (Roberts und Norman 1979, Merritt et al. 1986), kann für einen kombinierten Xylose-Absorptions $/ \mathrm{H}_{2}$-Exhalationstest beim Menschen (Casellas et al. 1993), bei der Katze (Muir et al. 1994) und beim adultem Pferd (Bracher et al. 1995b) verwendet werden.

In der vorliegenden Arbeit wurden diese 3 verschiedenen Zucker an gesunde saugende und mutterlose Fohlen verabreicht und die $\mathrm{H}_{2}$-Exhalation bestimmt.

\section{Material und Methoden}

Tiere

Zur Verfügung standen insgesamt 13 Fohlen $(7$ Welsh Ponies, 3 Vollblüter und 3 Halbblutfohlen), wobei einige Fohlen mehrmals untersucht wurden (Tab. 1). Das Alter der Fohlen reichte von 23-103 Tagen. 
Die Fohlen waren klinisch gesund und vertraut im Umgang mit Menschen. Die Untersuchungen wurden jeweils in der gewohnten Umgebung (in Boxen oder auf der Koppel) durchgeführt, wobei darauf geachtet wurde, daß die Fohlen ständigen Kontakt zu ihrer Mutter hatten und möglichst wenig Zwangsmaßnahmen bei den Manipulationen nötig waren.

Tab. 1: Signalement, Haltung und Verwendung der Fohlen

Identification, management and experimental use of the foals

\begin{tabular}{|r|c|c|c|c|c|l|}
\hline Nr & Name & Rasse & $\begin{array}{c}\text { Ge- } \\
\text { schlecht2 } \\
\text { Sex }\end{array}$ & mit Stute & $\begin{array}{c}\text { Alter } \\
\text { (Tage) }\end{array}$ & $\begin{array}{l}\text { Art der } \\
\text { Untersuchung } \\
\text { age } \\
\text { Type of } \\
\text { (days) }\end{array}$ \\
\hline 1 & SJ & WP & M & Ja & 39 & Xylose, Lactulose \\
\hline 2 & EB & WP & M & Ja & 67 & Xylose, Lactulose \\
\hline 3 & HP & WP & F & Ja & 91 & Xylose. Lactulose \\
\hline 4 & WN & WP & M & Nein & 85 & Xylose, Lactulose \\
\hline 5 & GR & WP & F & Nein & 23 & Xylose, Lactulose \\
\hline 6 & SQ & WP & M & Nein & 25 & Xylose, Lactulose \\
\hline 7 & LAP & VB & F & Ja & 43 & Lactulose \\
\hline 8 & NJA & VB & F & Ja & 83 & Lactulose \\
\hline 9 & MCR & VB & F & Ja & 28 & Laktose \\
\hline 10 & COL & VBC & M & Ja & 27 & Laktose \\
\hline 11 & VIR & VBC & F & Ja & 24 & Laktose \\
\hline 12 & NJA & VBC & M & Ja & 26 & Laktose \\
\hline 13 & CF & WP & F & Ja & 103 & Laktose \\
\hline
\end{tabular}

'WP=Welsh-Pony; $\mathrm{VB}=$ Vollblut, Thorougbred;

$\mathrm{VBC}=$ Vollblut/Thoroughbred-Cross

${ }^{2} \mathrm{~F}=$ weiblich, female; $\mathrm{M}=$ männlich, male

\section{Auffangen der Ausatmungs/uft}

Den Fohlen wurde eine Kleintier-Anästhesie-Maske (Eisenhut-Vet AG, Allschwil, $\mathrm{CH}$ ) über das Maul und die Nüstern gestülpt, die über einen 3-Weg-Hahn (Ambu-valve E, Cox Surgical Equipment, London, UK) mit einem 5 Liter-Atembeutel (Douglas bag, Plysu Protection Systems Ltd, Milton Keynes, UK) verbunden wurde (Abb. 1). Der Beutel wurde jeweils einmal gefüllt, unter manueller Kompression entleert und anschließend wieder mit der Maske verbunden. Nach der zweiten Füllung wurden der Beutel und die Maske abgesetzt und Atemluftproben in Duplikaten in Plastikspritzen (Omnifix, B.Braun, Melsungen, D) aufgezogen und mit Spritzenadaptern (Adapter Sarstedt, Nürnbrecht, D) verschlossen. Die Proben für die Bestimmung der $\mathrm{H}_{2}$-Konzentration wurden sofort oder innert 2 Stunden verarbeitet.

Die Fohlen tolerierten diese Prozedur problemlos und ließen sich nach einer kurzen Eingewöhnungszeit die Probenentnahme sogar im Liegen gefallen.

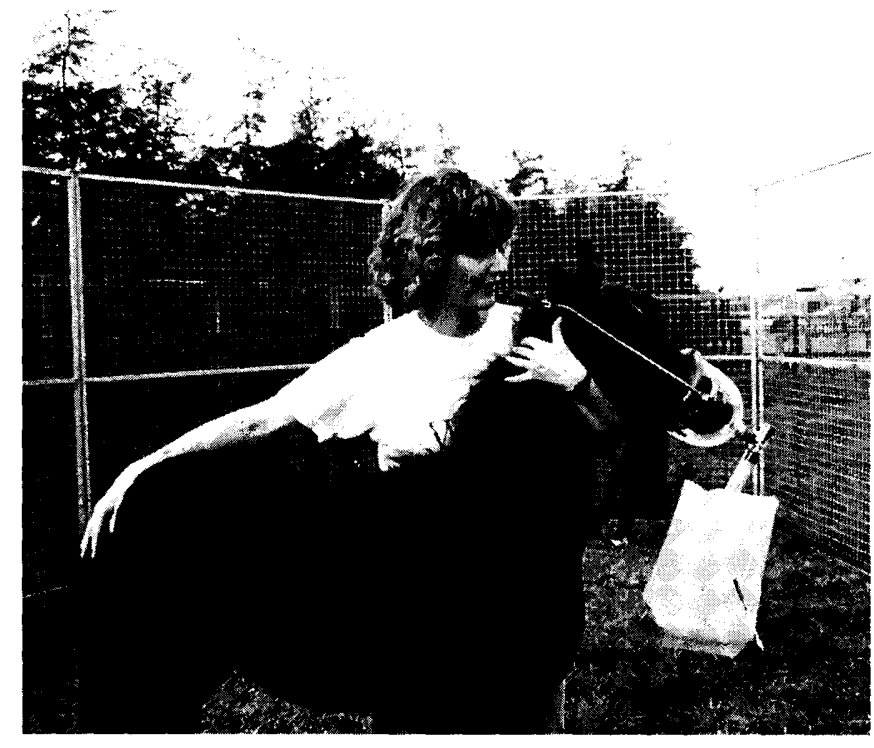

Abb. 1:Technik der Entnahme von Expirationsluft beim Fohlen Technique of breath collection in foals.

Dem Fohlen wird eine Kleintier-Anästhesiemaske über die Nase gestülpt. Mittels eines aufgesetzten Dreiwegventils kann die Ausatmungsluft in einem Atembeutel aufgefangen werden.

\section{Messung der Wasserstoff-Konzentration}

Die Messung der Wasserstoff-Konzentrationen erfolgte anhand einer elektrochemischen Methode in einem GMI Exhaled Breath Monitor (GMI Ltd., Renfrew, Scotland). Zu Beginn jeder Messreihe wurde eine Kalibration des Gerätes mit Hilfe eines Standardgases durchgeführt. Die Zuverlässigkeit dieser Methode wurde von Rumessen et al. (1987) untersucht und bestätigt.

\section{Plasma-Xylose Bestimmungen}

Bei 6 Tieren wurden Blutproben vor und in halbstündigen Intervallen während der folgenden 3 Stunden nach Xylose-Applikation entnommen. Blutproben wurden in OxalatFluorid-Röhren aufgefangen und sofort zentrifugiert und bei $-20^{\circ} \mathrm{C}$ eingefroren. Die Xylose-Bestimmung erfolgte nach der Parabromoanilin (PBA)-Methode von Roe und Rice (1948). Die Validierung dieser Methode beim Pferd erfolgte durch Roberts (1974a) und Roberts und Norman (1979).

\section{Verabreichung von Laktose, Lactulose und Xylose}

Bei den mutterlosen Fohlen wurde die Testsubstanz aufgelöst in der normalen Ration von Milchersatz morgens bei der ersten Fütterung per os verabreicht. Die Aufnahme war ungestört und innerhalb ca. 3 Minuten beendet. Danach hatten die Fohlen während der folgenden 4 Stunden keine weitere Fütterung, jedoch Zugang zur Stroheinstreu.

Bei den Saugfohlen wurde die Testsubstanz nach 3stündigem Fasten per Nasenschlundsonde verabreicht. Diese Fastendauer genügt, um die $\mathrm{H}_{2}$-Exhalation auf ca. 10 ppm zu senken (Bracher et al. 1995a). Während der folgenden 8 Stunden wurde der Maulkorb jeweils für 2-3 
Minuten nach jeder 2. Messung kurz abgenommen, um die Fohlen kurz bei der Mutter saugen zu lassen.

Bei allen Tieren wurden Ausatmungsluftproben vor und in halbstündigen Intervallen während 8 Stunden nach der Applikation entnommen. Bei der Verabreichung von Xylose wurden den Fohlen zusätzlich Blutproben vor und während 3 Stunden in halbstündigen Intervallen nach der Applikation entnommen. Dafür wurden intravenöse Verweilkatheter unter Lokalanästhesie in die Vena jugularis gesetzt. Das Blut wurde in Fluorid-Oxalat-Röhrchen aufgefangen und sofort zentrifugiert. Das Serum wurde zur späteren Xylose-Analyse eingefroren.

Die Substanzen wurden in den folgenden Dosierungen verwendet:

Laktose $\quad 1.0 \mathrm{~g} / \mathrm{kg} \mathrm{KM} \mathrm{D(+)-Laktose} \mathrm{Monohydrat} \mathrm{(Fluka}$ Biochemika, Buchs, $\mathrm{CH}$ ) in 20\%iger Lösung per Nasenschlundsonde oder in kommerziell erhältlichem Fohlenmilchersatz (mutterlose Fohlen).

Lactulose 0.5 bis $1.0 \mathrm{~g} / \mathrm{kg} \mathrm{KM}$ Lactulose (Rudolac ${ }^{\circledR}, 0.7$ mg Lactulose/ml Flüssigkeit, Streuli \& Co., Uznach, $\mathrm{CH}$ ) per Nasenschlundsonde oder in Milchersatz.

Xylose $\quad 0.5 \mathrm{~g} / \mathrm{kg} \mathrm{KM} \mathrm{D(+)-Xylose} \mathrm{(Fluka} \mathrm{Biochemika,}$ Buchs) als 10\%ige Lösung per Nasenschlundsonde oder in Milchersatz.

\section{Resultate}

\section{Laktose}

Bei allen 5 Fohlen wurde ein Anstieg der $\mathrm{H}_{2}$-Exhalation nach Laktose-Verabreichung festgestellt, wobei die Zeit bis zum Erreichen eines Peaks als auch die Höhe des Anstiegs stark variierte (Tab. 2 und Abb. 2). Beim ältesten untersuchten Fohlen CF (103 Tage alt) stieg die $\mathrm{H}_{2}$-Exhalation schon nach 30 Minuten an und sank im Laufe der achtstündigen Untersuchung wieder ab. Beim 28 Tage alten Fohlen MCR konnte ein dreigipfliger Verlauf mit ähnlichem ersten Anstieg beobachtet werden, während beim 24 Tage alten Fohlen VIR die $\mathrm{H}_{2}$-Exhalation erst gegen Ende der Untersuchungsperiode leicht anstieg.

\section{Lactulose}

Bei den 3 mutterlosen Fohlen im Alter zwischen 71 und 95 Tagen konnte kein Anstieg der $\mathrm{H}_{2}$-Exhalation nach Lactulose-Verabreichung beobachtet werden (Tab. 3 und Abb. 3). Auch bei dem ältesten der Saugfohlen (NJA) konnte mit Ausnahme der letzten Messung nach 8 Stunden kein Anstieg festgestellt werden. Bei den übrigen Saugfohlen wurden zum Teil sehr hohe $\mathrm{H}_{2}$-Exhalationen zwischen der 3. und 4. Stunde nach Verabreichung beobachtet.

\section{Xylose}

Die $\mathrm{H}_{2}$-Exhalation nach intragastraler bzw. oraler Verabreichung von Xylose schwankte ebenfalls beträchtlich sowohl bei den Saug- und den mutterlosen Fohlen. Bei den Saugfohlen wurde ein erster Peak zwischen 60 und 90 Minuten, und ein zweiter Peak bei 2 Tieren nach 5.5 und

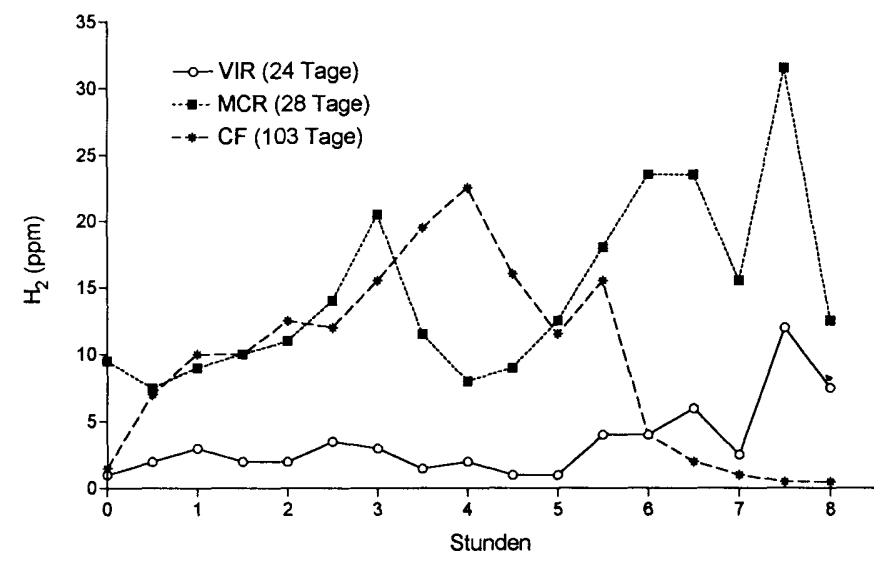

Abb. 2: $\mathrm{H}_{2}$-Exhalation nach Verabreichung von Laktose dargestellt bei 3 Saugfohlen im Alter von 24, 28 und 103 Tagen

$\mathrm{H}_{2}$-exhalation after administration of lactose in 3 sucking foals aged 24,28 and 103 days.

Tab. 2: $\mathrm{H}_{2}$-Exhalation bei 5 Saugfohlen unterschiedlichen Alters nach Verabreichung von $1.0 \mathrm{~g} / \mathrm{kg} \mathrm{KM}$ Laktose

$\mathrm{H}_{2}$-Exhalation in 5 sucking foals of different ages after administration of $1.0 \mathrm{~g} / \mathrm{kg}$ bwt. lactose

\begin{tabular}{|c|c|c|c|c|c|}
\hline $\begin{array}{l}\text { Zeit/time } \\
\text { Std//hrs }\end{array}$ & $\begin{array}{c}\text { VIR } \\
\text { (24 Tage) }\end{array}$ & $\begin{array}{r}\text { NJA } \\
\text { (26 Tage) }\end{array}$ & $\begin{array}{r}\text { COL } \\
\text { (27 Tage) }\end{array}$ & $\begin{array}{r}\text { MCR } \\
\text { (28 Tage) }\end{array}$ & $\begin{array}{r}\text { CF } \\
\text { (103 Tage) }\end{array}$ \\
\hline 0.0 & 1.0 & 10.5 & 19.0 & 9.5 & 1.5 \\
\hline 0.5 & 2.0 & 6.0 & 16.5 & 7.5 & 7.0 \\
\hline 1.0 & 3.0 & 8.5 & 20.0 & 9.0 & 10.0 \\
\hline 1.5 & 2.0 & 9.5 & 17.0 & 10.0 & 10.0 \\
\hline 2.0 & 2.0 & 9.5 & 10.0 & 11.0 & 12.5 \\
\hline 2.5 & 3.5 & 6.5 & 14.0 & 14.0 & 12.0 \\
\hline 3.0 & 3.0 & 11.0 & 10.0 & 20.5 & 15.5 \\
\hline 3.5 & 1.5 & 12.5 & 22.5 & 11.5 & 19.5 \\
\hline 4.0 & 2.0 & 18.5 & 9.0 & 8.0 & 22.5 \\
\hline 4.5 & 1.0 & 11.0 & 26.0 & 9.0 & 16.0 \\
\hline 5.0 & 1.0 & 21.5 & 20.5 & 12.5 & 11.5 \\
\hline 5.5 & 4.0 & 14.0 & 29.5 & 18.0 & 15.5 \\
\hline 6.0 & 4.0 & 18.0 & 29.5 & 23.5 & 4.0 \\
\hline 6.5 & 6.0 & 32.5 & 22.0 & 23.5 & 2.0 \\
\hline 7.0 & 2.5 & 25.0 & 28.0 & 15.5 & 1.0 \\
\hline 7.5 & 12.0 & 28.5 & 26.5 & 31.5 & 0.5 \\
\hline 8.0 & 7.5 & 26.5 & 31.0 & 12.5 & 0.5 \\
\hline
\end{tabular}

Der erste Anstieg von $>4 \mathrm{ppm}$ über den Ausgangswert ist fett markiert, die maximale $\mathrm{H}_{2}$-Exhalation ist fett und kursiv markiert.

The first rise of expiratory $\mathrm{H}_{2}>4 \mathrm{ppm}$ is shown in bold letters, whereas peak $\mathrm{H}_{2}$-exhalation is shown bold and italic.

6.5 Stunden erreicht (Abb. 4a). Sowohl bei den Saugfohlen als auch bei den mutterlosen Fohlen zeigte jeweils 1 Tier (EB und EE) eine deutlich höhere $\mathrm{H}_{2}$-Exhalation als die beiden anderen Fohlen der Gruppe.

Die Plasma-Xylose-Kurven sind in Abbildung $4 \mathrm{~b}$ darge- 
Tab. 3: $\mathrm{H}_{2}$-Exhalation bei 3 mutterlosen (of) und 5 Saugfohlen (sf)unterschiedlichen Alters nach Verabreichung von $1.0 \mathrm{~g} / \mathrm{kg} \mathrm{KM} \mathrm{Lactulose}$

$\mathrm{H}_{2}$-Exhalation in 3 orphan (of) and 5 sucking foals (sf) of different ages after administration of 1.0 $\mathrm{g} / \mathrm{kg}$ bwt. lactulose

\begin{tabular}{|c|c|c|c|c|c|c|c|c|}
\hline $\begin{array}{l}\text { Zeit/time } \\
\text { Std/hrs }\end{array}$ & $\begin{array}{r}S Q(71 \mathrm{Tg}) \\
(\mathrm{of})\end{array}$ & $\begin{array}{r}\text { GR }(95 \mathrm{Tg}) \\
(\mathrm{of})\end{array}$ & $\begin{array}{r}W N(89 \mathrm{Tg}) \\
\text { (of) }\end{array}$ & $\begin{array}{r}S J(32 \mathrm{Tg}) \\
\text { (sf) }\end{array}$ & $\begin{array}{r}E B(35 \mathrm{Tg}) \\
(\mathrm{sf})\end{array}$ & $\begin{array}{r}H P(49 \mathrm{Tg}) \\
(\mathrm{sf})\end{array}$ & $\begin{array}{r}\mathrm{NJA}(59 \mathrm{Tg}) \\
(\mathrm{sf})\end{array}$ & $\begin{array}{r}\operatorname{LAP}(43 \mathrm{Tg}) \\
(\mathrm{sf})\end{array}$ \\
\hline 0.0 & 1.5 & 2.5 & 1.5 & 6.5 & 13.5 & 7.5 & 32.5 & 7.5 \\
\hline 0.5 & 0.5 & 3.0 & 1.5 & 5.0 & 10.5 & 8.5 & 14.5 & 4.0 \\
\hline 1.0 & 0.0 & 3.5 & 1.0 & 10.0 & 11.0 & 7.0 & 16.0 & 8.5 \\
\hline 1.5 & 0.5 & 3.5 & 2.0 & 6.0 & 11.0 & 5.0 & 9.5 & 7.5 \\
\hline 2.0 & 2.0 & 7.0 & 4.5 & 7.5 & 9.5 & 4.0 & 5.5 & 9.0 \\
\hline 2.5 & 2.0 & 4.0 & 2.0 & 6.0 & 6.0 & 7.5 & 6.0 & 9.5 \\
\hline 3.0 & 1.5 & 3.5 & 4.0 & 6.5 & 12.5 & 5.0 & 6.0 & 11.5 \\
\hline 3.5 & 1.5 & 3.0 & 4.5 & & & & 6.5 & 16.5 \\
\hline 4.0 & & & & 21.5 & 20.0 & 13.5 & 5.5 & 28.5 \\
\hline 4.5 & 1.5 & 3.5 & 3.5 & & & & & \\
\hline 5.0 & & & & 17.5 & 54.0 & 11.5 & 7.5 & 27.0 \\
\hline 5.5 & 0.0 & 6.5 & 2.0 & & & & & \\
\hline 6.0 & & & & 30.0 & 80.0 & 18.5 & 7.5 & 35.0 \\
\hline 6.5 & 2.0 & 6.5 & 4.0 & & & & & \\
\hline 7.0 & & & & 19.5 & 83.0 & 17.0 & 5.5 & 43.0 \\
\hline 7.5 & 3.5 & 4.0 & 3.0 & & & & & \\
\hline 8.0 & & & & 29.0 & 118.0 & 10.0 & 14.0 & 53.0 \\
\hline
\end{tabular}

Der erste Anstieg von $>4$ ppm über den Ausgangswert ist fett markiert, die maximale $\mathrm{H}_{2}$-Exhalation ist fett und kursiv markiert.

The first rise of expiratory $\mathrm{H}_{2}>4$ ppm above basic value is shown in bold letters, whereas peak $\mathrm{H}_{2}$-exhalation is shown bold and italic.

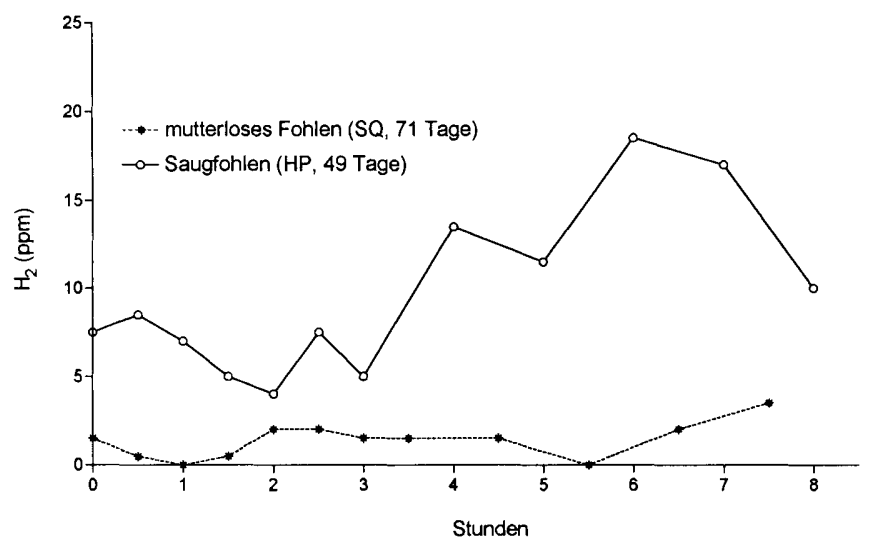

Abb. 3: $\mathrm{H}_{2}$-Exhalation nach Verabreichung von Lactulose dargestellt bei einem saugenden (HP) und einem mutterlosen Fohien (SQ). (Die Lactulose wurde dem Saugfohlen mit der Nasenschlundsonde verabreicht, während beim mutterlosen Fohlen die Verabreichung über die normale Morgenfütterung mit Milchersatz erfolgte.)

$\mathrm{H}_{2}$-exhalation after administration of lactulose in a sucking and an orphan foal. (lactulose was administred with nasogastric intubation after $3 \mathrm{hrs}$ of starvation in the sucking foal and mixed in the normal morning feed of milk replacer in the orphan foal). stellt: Während bei den Saugfohlen eine Maximal-Konzentration von $3.5 \mathrm{mmol} / \mathrm{l}$ innert 30 Minuten erreicht wurde, betrug die Maximalkonzentration bei den mutterlosen Fohlen nur $1.2 \mathrm{mmol} / \mathrm{l}$ und wurde erst 2-2.5 Stunden nach der oralen Applikation erreicht.

\section{Diskussion}

Bei gesunden Kindern werden nach der Aufnahme von Milch keine oder nur geringe Mengen an Wasserstoff mit der Ausatmungsluft ausgeschieden (Douwes et al. 1985). Bei frühgeborenen Säuglingen können jedoch bis zu 66\% der verabreichten Laktose das Kolon erreichen und zu entsprechend hohen $\mathrm{H}_{2}$-Exhalationskonzentrationen führen (MacLean und Fink 1980). Solche Säuglinge zeigen jedoch nicht unbedingt Symptome einer Laktose-Intoleranz und der fäkale Energieverlust nach Laktose-Verabreichung betrug bei diesen Kindern weniger als 15\%, was darauf hinweist, daß Laktose erfolgreich im Kolon zur Energiegewinnung fermentiert werden kann (MacLean und Fink 1980, Kien et al. 1987). Bei Fohlen im Alter von 20 bis 60 Tagen werden ebenfalls sehr hohe diurnale $\mathrm{H}_{2}$-Ex- 

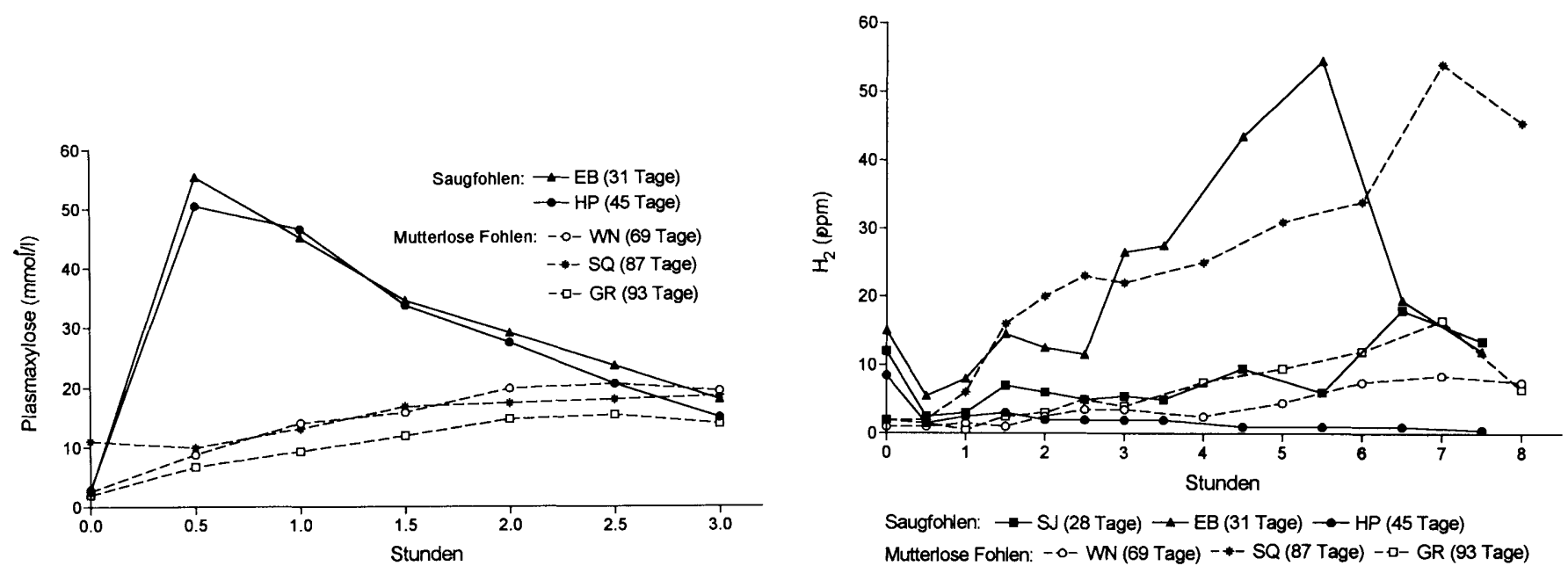

Abb. 4: $\mathrm{H}_{2}$-Exhalation (a) und Plasma-Xylose-Spiegel (b) nach Verabreichung von Xylose an 3 mutterlose und 3 Saugfohlen (aus: Bracher et al. 1995. Durchgezogene Linien: Fohlen mit Mutterstute, Verabreichung per Nasenschlundsonde; unterbrochene Linien: Mutterlose Fohlen, Verabreichung mit dem Milchersatz.)

$\mathrm{H}_{2}$-exhalation (a) and plasma xylose concentrations (b) after administration of xylose to 3 sucking and 3 orphan foals (full lines: sucking foals, administration of xylose with nasogastric intubation after 3 hrs of starvation; interrupted lines: orphan foals, administration of xylose mixed in the normal feed of milk replacer in the morning).

halationskonzentrationen gemessen, die nach 3stündigem Fasten stark absinken (Bracher et al. 1995a). Nach Verabreichung von $1 \mathrm{~g} / \mathrm{kg} \mathrm{KM}$ Laktose konnte bei allen untersuchten Fohlen ein Anstieg der $\mathrm{H}_{2}$-Exkretion beobachtet werden. Daraus wurde gefolgert, daß auch bei gesunden Fohlen ein Anteil der Laktose bis in das Zäkum und Kolon gelangt und dort einer bakteriellen Fermentation zugeführt wird, ohne daß dabei klinische Symptome einer Milchunverträglichkeit manifest werden.

Bei den Fohlen wurden häufig multiple Anstiege (Peaks) nach Substratverabreichung beobachtet. Interessanterweise beschrieb Cavell (1979) eine zweiphasige Magenentleerung bei frühgeborenen Säuglingen und Baker und Gerring (1994a) beobachtete mit Hilfe der PhenolRot-Verdünnungsmethode ebenfalls eine zweiphasige Magenentleerung beim Fohlen, was die früheren Beobachtungen von Alexander und Benzie (1951) bestätigt, daß die Magenentleerung schubweise mit dazwischenliegenden Pausen stattfindet.

Der kombinierte Xylose-Absorptions $/ \mathrm{H}_{2}$-Exhalationstest wird beim Menschen zur Abklärung einer Malabsorption empfohlen (Casellas et al. 1993). In der vorliegenden Arbeit wurde dieser Test auf zwei verschiedene Arten an den Fohlen durchgeführt: Bei den Saugfohlen wurde die Xylose wie in der Literatur angegeben (Merritt et al. 1986) per Nasenschlundsonde verabreicht, allerdings mit einer kürzeren Fastendauer, um einen negativen Effekt einer fasteninduzierten Hypoglykämie zu verhindern. Die Plasmaxylose-Verläufe entsprachen denjenigen von Merritt et al. (1986), was darauf hinweist, daß eine Fastendauer von 3 Stunden genügen sollte, um zuverlässige Resultate zu erhalten. Da die mutterlosen Fohlen problemlos alle Testsubstanzen mit inrer gewohnten Milchersatzration aufnahmen, wurde getestet, ob auch eine orale Xylose-Verabreichung möglich und damit das Setzen einer Nasen- schlundsonde vermeidbar war. Die Plasma-Xylose-Verläufe nach oraler Applikation zeigten jedoch einen viel langsameren und niedrigeren Anstieg, selbst im Vergleich zu den Referenzbereichen beim adulten Pferd (Roberts und Norman 1979, Freeman et al. 1989) und können deshalb kaum als Alternative zu einer Verabreichung per Nasenschlundsonde dienen. Der deutliche Unterschied zwischen den Applikationsarten beruht vermutlich auf einer verzögerten Magenentleerung infolge der Lipide oder anderer Bestandteile im Milchersatzpulver (Baker und Gerring 1994a). Die $\mathrm{H}_{2}$-Exhalationsprofile nach Xylose-Verabreichung waren in beiden Gruppen sehr variabel, was mit großen individuellen Unterschieden in der Magenentleerung beim Fohlen erklärt werden könnte, was auch schon zuvor bei Baker und Gerring (1994b) beobachtet worden war. Im Gegensatz zur Situation beim adulten Pferd (Bracher et al. 1995b) wurden bei den gesunden Tieren zum Teil deutliche Anstiege der $\mathrm{H}_{2}$-Exhalation, sowohl bei den Saug- als auch bei den mutterlosen Fohlen, beobachtet.

\section{Literatur}

Abrahamsson, H. (1988): Hydrogen breath test. In: An illustrated guide to gastrointestinal motility. Ed: D.Kumar und S. Gustavsson, Wiley, Chichester, pp. 125-130.

Alexander, F. und Benzie, D. (1951): A radiological study of the digestive tract of the foal. Q. J. Exp. Physiol. 36, 213-217.

Baker, S. J. und Gerring, E. L. (1994a): Gastric emptying of four liquid meals in pony foals. Res. Vet. Sci $56,164-169$.

Baker, S. J. und Gerring, E. L. (1994b): Gastric emptying of solid, non-digestible, radiopaque markers in ponies. Res. Vet. Sci 56 , 386-388. 
Bond, J. H. und Levitt, M. D. (1975): Investigation of small bowel transit time in man utilizing pulmonary hydrogen $\left(\mathrm{H}_{2}\right)$ measurements. J. Lab. Clin. Med. 85, 546-555.

Bracher, V., Ousey, J. C., Fazeli, A. R., Murgatroyd, P. und Rossdale, P. D. (1995a): Hydrogen breath test in foals. Biol. Reprod. Mono 1, 97-105.

Bracher, V., Steiger, R. und Huser, S. (1995b): Erste Erfahrungen mit dem kombinierten Xylose-Absorptions/Hydrogen-Exhalationstest beim Pferd. Schweiz. Arch. Tierheilk., 137, 297-305.

Bracher, V., Beatty, E. und Ousey, J. C. (1996): Postnatale Adaptation der Verdauung beim Fohlen. Pferdeheilkd. 12, 199-203

Calloway, D. H., Colasito, D. J. und Mathews, R. D. (1966): Gas produced by human intestinal microflora. Nature 212, 1238-1239.

Casellas, F., Chicharro, L. und Malagelada, J. R. (1993): Potential usefulness of hydrogen breath test with $D$-xylose in clinical management of intestinal malabsorbtion. Dig. Dis. Sci. 38, 321-327.

Cavell, B. (1979): Gastric emptying in preterm infants. Acta Paed. Scand. 68, 725-730.

Christl, S. U., Scheppach, W. und Kasper, H. (1995): Wasserstoffmetabolismus im Dickdarm-Physiologie und klinische Bedeutung. Z.Gastroenterol. 33, 408-413.

Douwes, A. C., Schaap, C. und van der Klei - van Moorsee, J.M. (1985): Hydrogen breath test in schoolchildren. Arch. Dis. Childhood 60, 333-337.

Freeman, D. E., Ferrante, P. L., Kronfeld, D. S. und Chalupa, W. (1989): Effect of food deprivation on D-xylose absorption test results in mares. Am. J. Vet. Res. 50, 1609-1612.

Kien, C. L., Liechty, E. A., Myerberg, D. Z. und Mullett, M. D. (1987): Dietary carbohydrate assimilation in the premature infant: evidence for a nutritionally significant bacterial ecosystem in the colon. Am.J.Clin.Nutr. 46, 456-460.

Levitt, M. D., French, P. und Donaldson, R. M. (1968): Use of hydrogen and methane excretion in the study of the intestinal flora. J.Lab.Clin.Med. 72, 988-989.

MacLean, W. C. und Fink, B. B. (1980): Lactose malabsorption by premature infants: magnitude and clinical significance. J. Pediatr. 97, 383-388.

Merritt, T., Mallonée, P. G. und Merritt, A. M. (1986): D-xylose absorption in the growing foal. Equine Vet. J. 18, 298-300.

Metz, G., Jenkins, D. J. A. und Peters, J. J. (1975): Breath hydrogen as a diagnostic method for hypolactasia. Lancet $i$, 1155-1157.

Muir, P., Gruffydd-Jones, T. J., Cripps, P. J., Papasouliotis, K. und Brown, P. J. (1994): Breath hydrogen excretion after oral administration of xylose to cats. J. Small Anim. Pract. 35, 86-92.

Roberts, M. C. (1974): The $\mathrm{D}(+)$ xylose Absorbtion test in the horse. Equine Vet. J. 6, 28-30.
Roberts, M. C. und Norman, P. (1979): A re-evaluation of the $\mathrm{D}(+) \times y$ lose absorption test in the horse. Equine Vet. J. 11 , 239-243.

Roe, J. H. und Rice, E. W. (1948): A photometric method for the determination of free pentoses in animal tissue. J.Biol.Chem. 173, 507-512.

Rumessen, J. J., Kokholm, G. und Gudmand-Høyer, E. (1987): Methodological aspects of breath hydrogen $\left(\mathrm{H}_{2}\right)$ analysis. Evaluation of a $\mathrm{H}_{2}$ monitor and interpretation of the breath $\mathrm{H}_{2}$ test. Scand. J. Clin. Lab. Invest. 47, 555-560.

Zentek, J. (1992): Mikrobielle Gasbildung im Intestinaltrakt von Monogastriern Teil II: Pulmonale Ausscheidung von Wasserstoff und Methan. Übers. Tierernährg. 20, 91-122.

Verena Bracher

Klinik für Wiederkäuer- und Pferdemedizin

Departement für Innere Medizin

Veterinärmedizinische Fakultät der Universität Zürich

Winterthurerstr.260

$\mathrm{CH}-8057$ Zürich

Schweiz

P. D. Rossdale

Jennifer C. Ousey

Beaufort Cottage Stables

High Street

Newmarket

Suffolk $C B 89 B H$

UK

W. R. Allen

TBA Equine Fertility Unit

Mertoun Paddocks

Woodditton Road

Newmarket

Suffolk $C B 89 B H$

UK

Emily Beatty

Dunn Clinical Nutrition Laboratories

Addenbrookes Hospital

Cambridge

UK 